\title{
Creating a New Dynamic Measure of the Useful Field of View Using Gaze- Contingent Displays
}

\author{
Ryan V. Ringer ${ }^{*}$ \\ Aaron P. Johnson ${ }^{* *}$ \\ Kansas State \\ University \\ Concordia \\ University
}

John G. Gaspar ${ }^{* * *}$

\author{
University of \\ Illinois, Urbana- \\ Champaign, \\ Beckman Institute
}

\author{
Mark B. Neider ${ }^{* * * *}$
}

University of
Central Florida

\author{
James Crowell ${ }^{\dagger} \quad$ Lester C. Loschky \\ Arthur F. Kramer \\ University of \\ Illinois, Urbana- \\ Champaign, \\ Beckman Institute
}

\begin{abstract}
We have developed a measure of transient changes in the useful field of view (UFOV) in simulators using gaze-contingent displays (GCDs). It can be used to evaluate safety-critical tasks such as driving or flight, and in training to increase the UFOV under cognitive load, stress, and fatigue. Unlike the established UFOVC measure, our measure can be used in simulators. Furthermore, previous peripheral detection tasks used in simulators controlled neither the target's retinal eccentricity nor stimulus intensity. Our approach overcomes these limitations by using GCDs to present stimuli producing equal performance across eccentricities under single-task conditions for two dependent measures: blur detection and Gabor orientation discrimination. We then measure attention under dual task conditions by varying cognitive load via an $\mathrm{N}$ back task. Our results showed blur sensitivity varied predictably with retinal eccentricity, but detection of blur did not vary with cognitive load. Conversely, peripheral Gabor orientation discrimination showed a significant cognitive load decrement. While this method is still in development, the results suggest that a GC UFOV method is promising.
\end{abstract}

CR Categories: H.5.2 [Information Interfaces and Presentation]: User Interfaces - User-centered Design, Windowing Systems, Screen Design, Evaluation/Methodology; I.4.2 [Image Processing and Computer Vision]: Compression (Coding); General Terms: Human Factors, Experimentation.

Keywords: attention, useful field of view, UFOV, functional field of view, FFOV, gaze-contingent displays, multiresolutional displays, dual-resolution displays, Gabor orientation discrimination, eyetracking, eye movements, visual perception, peripheral vision, simulators

\section{Measures of the Useful Field of View}

\author{
*e-mail: rvringer@k-state.edu \\ **e-mail: aaron.johnson@concordia.ca \\ ***e-mail: jgaspar2@illinois.edu \\ ****e-mail: Mark.Neider@ucf.edu \\ ${ }^{\dagger}$-mail: jimc@illinois.edu, ${ }^{\ddagger}$ a-kramer@illinois.edu \\ §e-mail: loschky@k-state.edu
}

The useful field of view (UFOV) is an important theoretical construct that can be used to describe situational awareness. "The UFOV is the total visual field area in which useful information can be acquired without eye and head movements (i.e., within one eye fixation)" [Ball, et al. 1988]. Therefore, it is a measure of speed of processing and attentional breadth. Agiven individual's UFOV can vary from moment-to-moment as a function of workload [Atchley and Dressel 2004; Ball, et al. 1988; Crundall, et al. 2002; Recarte and Nunes 2003; Williams 1985], and can increase with training [Ball, et al. 1988; Roenker, et al. 2003]. The UFOV(C has been shown to reliably predict negative outcomes in real world tasks, such as traffic accidents during driving [Ball, et al. in press; Clay, et al. 2005], most likely because people with a narrow UFOV often fail to perceive safety-critical information in their environment. Thus, the UFOV can be thought of as a critical determinant of a person's situational awareness [Endsley 1995].

Our particular interest is in creating a dynamic contextualized measure of transient changes in the UFOV that can be used in driving simulators, flight simulators, and virtual reality. Being able to study such situation-dependent moment-to-moment changes in a person's UFOV can be extremely useful in highlighting the specific dangers caused by a narrow UFOV, and thus suggest ways to either avoid or counteract those dangers, for example through training. However, the best-known measure of the UFOV (the UFOVC test developed by Visual Awareness Research Group, Inc.) is a standardized measure that uses a static array of briefly flashed simple stimuli. Clearly, superimposing such a stimulus over a competing stimulus, such as the windscreen of a driving or flight simulator would be problematic. Specifically, the presentation of the UFOV array would likely mask the competing stimulus and disrupt performance in the primary (simulated) task. Perhaps even more importantly, because the UFOVC involves both detection and localization responses [Ball, et al. 1988; Ball, et al. 1990], it would likely cause severe dual-task interference with dynamic tasks that are inherently spatial, such as simulated driving or flight. For example, having to suddenly point to the location of a UFOV target would likely interfere with the primary task. So, while the commercial UFOV C test might provide an ad hoc predictor of future performance, it cannot provide a momentby-moment account of how an individual's UFOV changes based on the work load, stress, and demands associated with performing the actual task of interest.

There are, however, other measures of the UFOV that have been used in dynamic task-specific contexts, such as the Peripheral Detection Task (PDT). The PDT has been used to measure moment-to-moment changes in UFOV as a function of workload [Crundall, et al. 2002; Jahn, et al. 2005]. Such measures have generally not required the participant to either localize or identify 
the target, but simply to detect it, thus causing far less dual-task interference with the primary task, such as driving [Crundall, et al. 2002; Jahn, et al. 2005]. However, there are important limitations in how the PDT has been implemented. Chief among these are that 1) the targets have fixed physical locations, making it impossible to precisely control retinal eccentricity of the targets from moment-to-moment, and 2) the targets have fixed intensities, thus confounding the effects of eccentricity-dependent contrast sensitivity or resolution and attentional breadth. For example, the PDT paradigm, as it has previously been implemented in driving simulators, has used target stimuli, such as LEDs, with physically fixed locations and fixed stimulus intensities [Jahn, et al. 2005]. Similarly, in Crundall, et al.'s [2002] study, targets were in a cross configuration, located above, below, and to the left and right of the center of the screen, again with fixed stimulus intensities. Thus, our proposed framework described below overcomes all of the above-noted limitations in the UFOVC and previous instantiations of the PDT. Although the issue of determining a suitable cognitive load level capable of inflicting an optimal degree of UFOV impairment is still one of contention, this text addresses a number of considerations relevant to the implementation of this novel framework.

\section{Proposed Framework: Gaze-Contingent Atten- tion Measures For Simulated Environments}

Thus far, there has been a trade-off between ecological validity and experimenter control within the current measures of the UFOV. Here, we propose a framework for measuring the UFOV in realistic simulated environments while greatly improving stimulus control for our dependent measures of attentional breadth.

A first critical element of our framework is using gaze-contingent displays [Duchowski, et al. 2004; Loschky and McConkie 2002, 2000; McConkie and Rayner 1976, 1975; Reingold, et al. 2003]. This allows us to measure attention across the field of view on any given eye fixation during dynamic viewing. In our framework, the attentional targets are presented gaze-contingently on occasional fixations [Loschky, et al. 2005; Loschky and Wolverton 2007]. In this way, the viewer has a chance to make a response to the gaze-contingent target before the next target is presented. Furthermore, by presenting the targets only occasionally, the viewer can focus most of their attention on the primary task (e.g., simulated driving) as well as the cognitive load task. Thus, targets are only presented every Nth fixation. Furthermore, half of the presentations should be "catch trials" in which either no target is presented (e.g., no blur for a blur detection task) or the standard stimulus is presented (e.g., a vertical Gabor patch for a Gabor discrimination task).

In addition, for gaze-contingent presentation of stimuli, it is important that the speed of updates at the end of each saccade are made quickly enough to avoid disrupting perception or capturing attention [Reingold, et al. 2003]. Gaze-contingent blur update delays of $60 \mathrm{~ms}$ or less do not increase the detectability of blur [Loschky, et al. 2007]. To determine the update delay, it is best to use an artificial eye and empirically measure the delay between the artificial eye movement and the display change. [For a detailed discussion of issues related to update delays see Loschky, et al. 2007; Reingold, et al. 2003.]].

A second critical element in the framework is the dependent measure of attention. These are generally detection tasks or discrimination tasks, both of which have been widely used to measure attention. We have implemented our framework using blur detection and Gabor orientation discrimination, but many other dependent measures are possible (e.g., detection or discrimination of color [Duchowski, et al. 2009], motion, etc.). The dependent measures can be generally applicable to any scene (as blur detection and Gabor orientation discrimination are), or have specific content that is context-dependent (e.g., traffic or pedestrian incursions, enemy combatants, etc.).

A third critical element of our framework is to manipulate available attentional resources and determine the effects on our dependent measures. In our framework, attentional resources can be manipulated in numerous ways. In our implementation of the framework we have used the N-back to vary cognitive load, because it is well-respected, widely used, and the load can be varied incrementally [Cohen, et al. 1997; Jaeggi, et al. 2010; Kane, et al. 2007; Owen, et al. 2005]. We can then compare performance in single-task versus dual-task conditions. Manipulations of attentional resources can be generally applicable (such as dual-tasks, fatigue, stress, or drugs), or context-dependent (e.g., traffic density, or hazard detection in driving simulators).

The fourth critical element of our framework is to factor out eccentricity-dependent changes in vision that are independent of attention (e.g., the loss of acuity with increasing eccentricity). We can do this by first determining each viewer's eccentricitydependent perceptual thresholds (for the given dependent measure) under single-task conditions. Then, we can compare performance for single-task and dual-task conditions at each eccentricity to determine the effects of dual-task cognitive load on the useful field of view. The estimation of baseline eccentricity-dependent thresholds can be measured prior to running the main experiment. This allows one to then use fixed, thresholded, target intensities during the experiment, and measure changes in sensitivity (e.g., d') to them. Alternatively, one can estimate eccentricitydependent thresholds during the experiment, and use those thresholds as the dependent measure of attention.

\subsection{How Attention Should Influence our Measures}

Research has shown that attending to a region of space can improve signal strength for that region in space by increasing contrast and spatial resolution [Carrasco 2011]. In addition, Carrasco and colleagues have argued that attention increases the signal strength of a given stimulus. Conversely, as attention is focused on a particular stimulus or region, we can often become less sensitive or unaware of changes in the rest of the image.

In the general framework, we propose that attention will change sensitivity to detection or discrimination tasks (e.g., blur detection or orientation discrimination) across the visual field, and thus can be used as a measure of UFOV. Under low load conditions, attention should be evenly distributed across the visual field, but should show changes in threshold with eccentricity. However, under high load conditions, attention will be more limited, thus reducing performance in the blur detection and orientation discrimination tasks. The decrease in performance due to changes in attention should follow one of two possible patterns, either "tunnel vision" or "general interference" [Crundall, et al. 2002; Williams 1988]. Tunnel vision should show attention narrowly focused on the point of fixation. Thus, participants should be less sensitive to changes in blur and orientation as you move from the point of fixation to greater eccentricities, leading to increased thresholds. Conversely, general interference should show an equal decrement at all retinal eccentricities, from central vision to the visual periphery. In order to distinguish between these two possible patterns of attention across the visual field, as noted earlier, we must factor out eccentricity-dependent changes in vision 
that are independent of attention, by measuring each viewer's eccentricity-dependent perceptual thresholds (e.g., for either blur detection or orientation discrimination) under single-task conditions. By comparing single-task versus dual-task performance at each eccentricity we determine whether cognitive load produces tunnel vision or general interference.

Below, we will describe the general methods for implementing our framework, along with more specific implementations for specific dependent measures. We will then briefly report on the results of several experiments using these two dependent measures, and discuss the suitability of each as valid measures of attentional breadth within our framework.

\section{Methods}

\subsection{Blur Detection}

Blur is a natural part of vision, and occurs due to changes in focal length, distance between objects in depth, motion, and various vision problems such as refractive errors (e.g., myopia, hyperopia, or astigmatism). Sensitivity to blur decreases with retinal eccentricity. Yet, the fact that you cannot perceive fine details in your visual periphery, based on the rapid drop-off of contrast sensitivity with increasing retinal eccentricity, is generally not perceived as "blur" nor does it result in accommodation [Wang and Ciuffreda 2004]. On the other hand, when the degree of image blurring exceeds a threshold, such that the highest spatial frequencies removed would have been potentially resolvable, then blur is detected [Loschky, et al. 2005].

\subsection{Gabor Orientation Discrimination}

Humans show a high degree of orientation selectivity, with thresholds for discriminating changes in orientation being typically 1 degree [Burr and Wijesundra 1991; Webster, et al. 1990]. This is in spite of the evidence that neurons in the visual cortex show board orientation tuning functions [De Valois and De Valois 1988]. Orientation thresholds typically increase with retinal eccentricitiy [Mareschal and Shapley 2004].

\section{$4 \quad$ Blur Detection Measure}

\subsection{Methods}

\subsubsection{Experiment Overview}

Within a trial, participants carried out three different tasks: 1) Memorization of the scene image, for a later (relatively easy) picture recognition task. This was done to encourage participants to actively explore the image with many eye movements, (with the recognition memory test given at the end of each session). 2) Blur detection in the image, which only occurred occasionally for single fixations. Blur levels were varied across eccentricity, and thresholds were calculated using an adaptive threshold estimation algorithm. 3) A cognitive load task (N-back), which varied in terms of the load from block to block. In order to counterbalance the effects of learning and fatigue throughout the experiment, participants completed the blur detection task through six sessions. Each session contained all levels of the cognitive load manipulation, while the order of cognitive load blocks was counterbalanced using a Latin square matrix.

\subsubsection{Participants}

Three lab members (two females) with normal vision (20/30 or better) volunteered to participate as subjects in the experiment. Each session lasted 1.5-2 hours, with the entire experiment requiring 9-12 hours.

\subsubsection{Apparatus}

Blur detection sessions were conducted on a custom built Origin Genesis PC running Microsoft Windows 7, with an Intel Core i7 970 processor $(3.2 \mathrm{GHz})$, with $24 \mathrm{~GB}$ DDR3 RAM, and a $2 \mathrm{~GB}$ Radeon HD6950 video card. Stimuli were presented on a ViewSonic Graphics Series 19" CRT monitor (Model G90fb) at $85 \mathrm{~Hz}$ refresh rate, and at a screen resolution of $1024 \times 768$ pixels. A chin rest was used to stabilize head position at $60.33 \mathrm{~cm}$ away from the screen. This provided a viewing angle of $33.67^{\circ} \times 25.50^{\circ}$ for all images. The monitor display was calibrated with a Spyder3Elite photometer with a maximum and minimum luminance of $91.3 \mathrm{~cd} / \mathrm{m} 2$ and $.33 \mathrm{~cd} / \mathrm{m} 2$, respectively, and a gamma of 2.21 .

Eye position was acquired non-invasively using a video-based eyetracker (EyeLink 1000/2K, SR Research, Ottawa, Ontario). The EyeLink system recorded monocular eye position with a sampling resolution of $1,000 \mathrm{~Hz}$. Given the unknown, but expected delay between an actual eye movement and the update of the stimulus on the screen, gaze-contingent display change latency was measured using an artificial eye (see Appendix A in Bernard et al. 2007 for more details on the concept on an artificial eye). We found that the measured latency in our apparatus ranged between 18.25 and $22.25 \mathrm{~ms}$ (Mean $=20 \mathrm{~ms}, 95 \%$ confidence interval of $19.75-20.25 \mathrm{~ms})$. This is under the $80 \mathrm{~ms}$ latency that has been shown to first produce increased gaze-contingent image blur detection rates [Loschky, et al. 2007]. Consequently, the gazecontingent display updates should not have increased the detectability of blur by our participants.

\subsubsection{Stimuli}

We used a total of 1,296 images from the SUN image database [Xiao, et al. 2010]. The images were comprised of a large number of scene categories, including forests, mountains, street scenes, and home and building interiors. Images were excluded if they contained poor image focus, predominantly low-frequency information, or watermarks. All images were $1024 \times 768$ pixels.

To determine the degree to which subjects could detect blur on specific eye fixations, we used an occasional gaze-contingent blur detection task [Loschky, et al. 2005; Loschky, et al. 2007]. In this task, we used a gaze-contingent bi-resolution display, in which images were presented with two levels of resolution, a circle of high resolution imagery surrounded by lower resolution imagery, with the center of high resolution placed at the center of gaze (Fig. 1) using eyetracking [Duchowski, et al. 2004; Loschky, et al. 2002; Reingold, et al. 2003]. We used a Gaussian low-pass filter, which has previously been shown to have linear detection properties [Murray and Bex 2010]. The Gaussian low-pass filters were defined by:

$$
A_{(f)}=E\left\lfloor\frac{f^{2}}{2 \times S D^{2}}\right\rfloor
$$

where $f$ is the spatial frequency, and SD is the standard deviation of the Gaussian. Using Mathworks MatLab (2012a), we then generated 450 blurred versions of the each base image, with a low-pass filter spatial frequency cut-off that ranged from a maxi- 
mum of 50 cycles per degree (cpd; the human limit of resolution) and a minimum of $0.50 \mathrm{cpd}$ (highly blurred). These images were then used as windowed masks to be presented during a valid blur trial. In order to reduce the saliency of any edges between the blurred and unblurred region at the window's edge, the strength of blur was tapered.

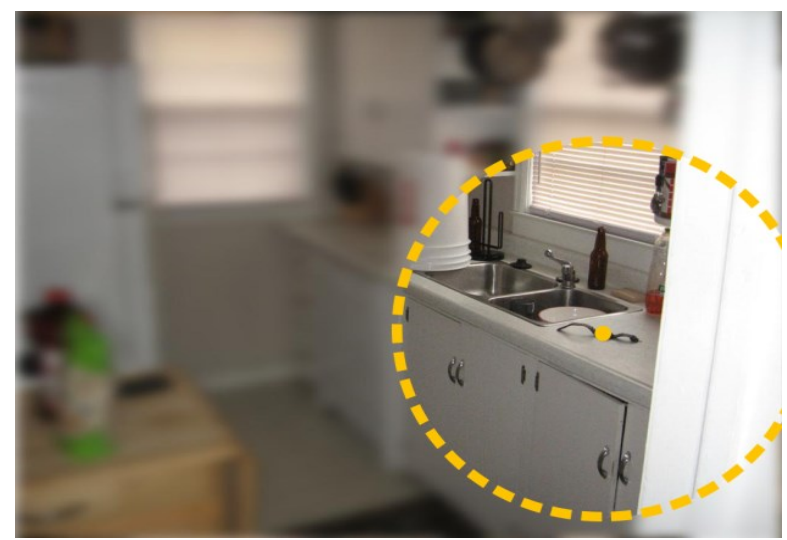

Figure 1. Sample image of a $9^{\circ}$ eccentricity blurred image. For ease of illustration, the blurred edge is highlighted with the yellow dotted circle and the viewer's point of fixation is indicated with a central dot, though these were not visible to the participants.

\subsubsection{Procedure}

\subsubsection{Blur Detection Task}

We used a go-no-go task to test viewers' ability to detect gazecontingent blur. For purposes of signal detection analyses, we presented an equal number of blur-present and blur-absent displays, which occurred on every $7^{\text {th }}$ fixation, with the order of blurpresent/absent displays randomized to prevent participants from detecting patterns in the displays. On blur-present displays, we presented the blur for a single eye fixation, with the normal unaltered image shown on the majority of fixations (13 out of every 14). Participants were asked to press a mouse button with their right hand any time they detected blur [Loschky, et al. 2005; Loschky, et al. 2007]. During blur-absent displays (i.e., catch trials), an identical copy of the original unaltered image was presented for the same duration. Following each presentation, participants had up until the next blur-present/absent display (i.e., 7 fixations) to make a go-response, otherwise it as coded as a no-go response. (See example video of the blur detection task here.)

Blur was presented at one of four retinal eccentricities $\left(0^{\circ}, 3^{\circ}, 6^{\circ}\right.$ and $9^{\circ}$ ), outside of a gaze-contingent circular window, with blur values being controlled by an adaptive threshold estimation procedure. Inside the window was unaltered imagery. Each trial ended when the participant had made 56 fixations ( 1 presentation/7 fixations x 2 blur-present/absent displays $x 4$ eccentricities). Order of eccentricities was randomized for each trial/image. To determine blur thresholds, we used the Single Interval Adjustment Matrix (SIAM) adaptive threshold estimation procedure [Kaernbach 1990], which is suited to a "go/no-go" task. We simultaneously estimated blur thresholds for each eccentricity on a per-block basis, with the estimations being continuously updated across all images within a block.

\subsubsection{Cognitive Load Manipulation}

We used an N-back go-no-go task using auditory presentations of letter targets. The task requires a participant to hold a list of nitems in working memory, to check if the most recently presented item is the same as the item presented n-items back in the list. If an item was valid, participants were instructed to make a goresponse on a game controller. The difficulty of the task increases as a function of the $n$ value of the task [Cohen, et al. 1997; Jaeggi, et al. 2010]. The easiest N-back level was 0-back, in which subjects simply had to respond whenever the letter " $m$ " was presented. Letters were presented every $2000 \mathrm{~ms}$, and the mean presentation duration for each item was approximately $630 \mathrm{~ms}$. (See example video of the dual N-back + blur detection task here.)

In addition to $0,1,2$, and 3-back tasks, we also included two control conditions: 1) The single task with n-back letters presented while participants were instructed to ignore them, and 2) the single-task with no n-back letters. N-back performance feedback $(\%$ correct) was given after every 6 images to ensure that participants were sufficiently engaged in the cognitive load task. Importantly, no feedback was given on the blur detection task so that the nback task was given implicit priority.

\subsection{Results}

Due to the small number of participants and large number of observations for each subject, we used a Restricted Estimate of Maximum Likelihood [REML Kenward and Roger 1997] to assess fixed effects of cognitive load level for the n-back task, and fixed effects of cognitive load and eccentricity in the blur detection task. Each subject was considered a random variable in the analysis.

\subsubsection{N-Back Performance}

The N-back results showed that subjects indeed were less sensitive in the N-back task as $n$ increased from 0 to 3 -back $(F(3,6)=$ $10.371, p=.0076)$. This was as expected, and showed that the Nback task was indeed capable of creating a cognitive load for our subjects.

\subsubsection{Blur Detection Performance}

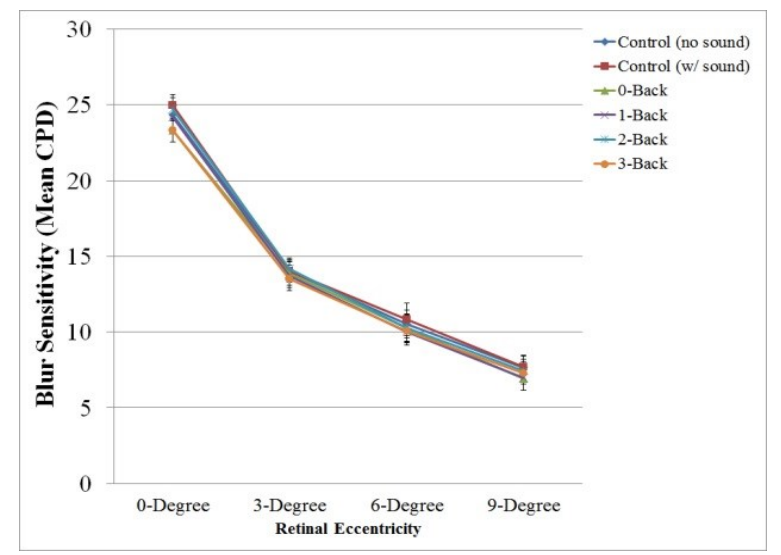

Figure 2. Blur detection results as a function of retinal eccentricity and cognitive load.

As shown in Figure 2, the blur detection task showed the expected significant main effect of eccentricity, indicating that our estima- 
tion of blur thresholds was, in fact, sensitive to differences in spatial resolution as a function of eccentricity $(F(1,1.661)=$ $3930.56, p=.0008$ ). However, as shown in Figure 3, we failed to find a significant main effect of cognitive load $(F(5,8.181)=$ $2.248, p=.146)$, nor was there a significant interaction between cognitive load and eccentricity $(F(5,6.98)=1.778, p=.236)$.

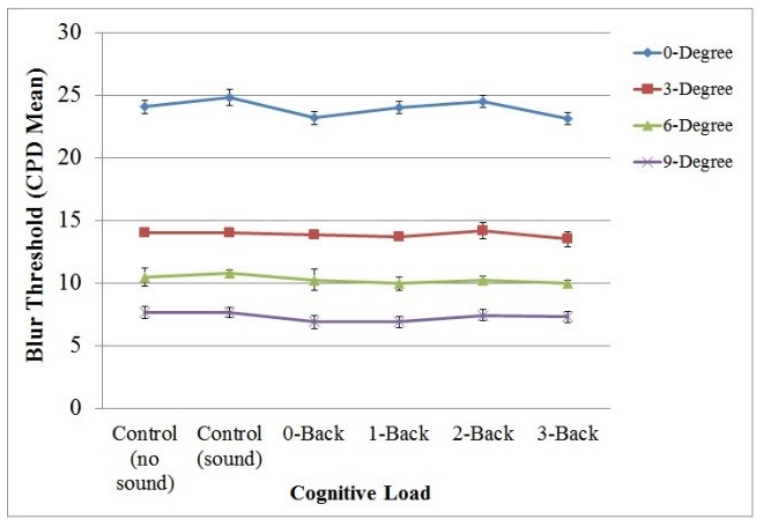

Figure 3. The same blur detection results as shown in Figure 2, but plotted as a function of cognitive load and eccentricity.

\subsection{Discussion}

The results surprisingly suggest that blur detection may be unaffected by cognitive load, and thus may not be a suitable measure of attention within our general framework. We therefore conducted a second experiment (with different subjects) in which we tested the hypothesis that the failure of the N-back task cognitive load to interfere with our visual attention task was due to the auditory modality of the N-back. In that experiment, we used a visual $\mathrm{N}$-back task, but found nearly identical results to the auditory nback task. We also carried out a third experiment (with different subjects) in which we tested the hypothesis that the failure to show an effect of the N-back cognitive load on our measure of attention (blur detection) was due to attentional capture by the gaze-contingent onset of the blur (a "pop-out" effect). In that experiment, we used a tachistoscopic version of the blur detection task, in which target images were rapidly flashed, and blur was an integral part of the target images. However, we again we found nearly identical results to the previous experiments. Thus, we conclude that blur detection either occurs preattentively, or simply has a very high threshold for being affected by cognitive load[Loschky, et al. in press]. In either case, it seems that blur detection is not well suited as a measure of the UFOV.

\section{Gabor Orientation Discrimination Measure}

\section{$5.1 \quad$ Methods}

\subsubsection{Experiment Overview}

Based on the above results using the blur detection task as our dependent measure of attention, we decided to implement our general framework with an alternative dependent measure, Gabor orientation discrimination. Within our framework, a primary advantage of discrimination tasks over detection tasks is that they are more sensitive to attentional manipulations [Carrasco 2011; Correa, et al. 2004; Kowler, et al. 1995]. Gabor orientation discrimination tasks, in particular, have shown clear effects of attentional manipulations in spatial cueing tasks. However, the above studies using Gabor discrimination as a dependent measure of attention all used neutral gray backgrounds, rather than Gabor patches embedded in real-world scenes. Additionally, those studies did not manipulate attention through cognitive load, nor did they present the Gabor patch targets gaze contingently. Thus our study is, to our knowledge, the first to employ such a method. The experiment using Gabor orientation discrimination also differed from our previous experiments in two key ways. First, instead of using a free-viewing picture memorization task, participants performed a driving task in which they were instructed to follow a lead vehicle in a medium fidelity simulator. Second, responses to the n-back task were spoken, rather than made with a mouse response.

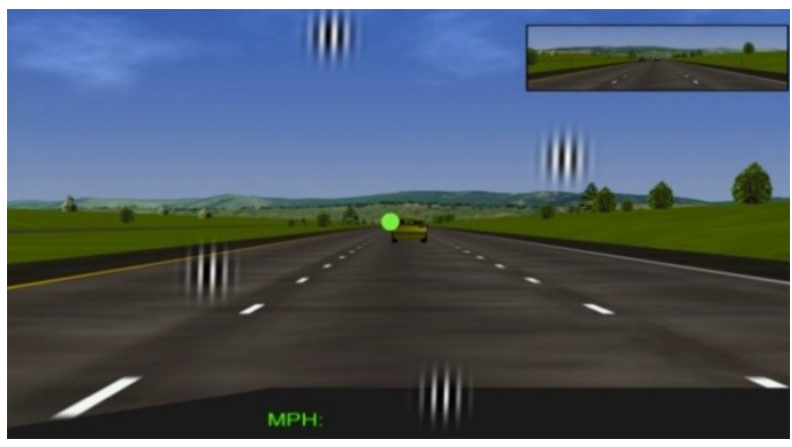

Figure 4. Still image of the Gabor orientation discrimination task in the driving simulator. This shows a valid (non-vertical Gabor patch) trial. The central green dot indicates the viewer's point of fixation, but was not visible to participants.

\subsubsection{Participants}

Twelve young adults with valid driver's licenses and normal (20/30 or better) vision were recruited as paid subjects at a research institute separate from Experiment 1.

\subsubsection{Apparatus}

The bulk of the experiment used a desktop driving simulator running Hyperdrive software (by DriveSafety ${ }^{\mathrm{TM}}$ ). All data was collected through the HyperDrive system via a desk-mounted Logitech steering wheel and under-desk pedals, at $60 \mathrm{~Hz}$. A SmartEye Pro 5 gaze tracker was also used. This system uses four stationary IR cameras to reconstruct head pose and gaze position at the same sample rate as the HyperDrive. Voice recording of Nback responses were recorded through an EasyVR speech recognition module.

We used an overlay rendering PC to embed our gaze-contingently presented Gabor patch targets within the dynamically simulated driving scenes. This PC accepted two inputs: a) the video output from the HyperDrive system using an Epiphan VGA2PCIe capture card, and b) the gaze position from the SmartEye via UDP. A Python program collected the inputs, performed fixation identification, decided when to render a stimulus overlay based on experimental parameters, and used OpenGL Shader Language (GLSL) and an NVidia GeForce GTX 480 GPU to render the HyperDrive input image with or without a stimulus overlay. Its output was displayed on an 80-inch Sony Aquos LED monitor (Figure 1) at $60 \mathrm{fps}$ and $1024 \times 768$ resolution.

\subsubsection{Stimuli}

Environments and vehicles were created using Hyperdrive's standard tiles and entities, and ambient traffic was controlled with 
customized Tcl/Tk scripts. The display computed a realistic driving dynamic, and rendered the visible scene at $60 \mathrm{fps}$ and $1024 \times 768$ resolution.

\subsubsection{Procedure}

\subsubsection{Gabor Orientation Discrimination Task}

Prior to the main experiment, participants were pre-tested to determine their Gabor orientation discrimination threshold that would produce $80 \%$ accuracy at each retinal eccentricity $\left(10^{\circ}\right.$ or $\left.15^{\circ}\right)$. The absolute value of the Gabor's deviation from the vertical axis varied based on the participants' responses using the PSI method [Kontsevich and Tyler 1999]. The orientation values that were determined during this pre-test phase were used in the testing phase.

During the testing phase, a set of four Gabor patches, each equidistant from fixation, were occasionally presented at either $10^{\circ}$ or $15^{\circ}$ from the point of fixation (Fig. 4). The Gabors were smoothly blended with the image frames, with each patch's background value being set to the mean brightness of the region under each patch. Participants were required to make right/left orientation distinctions by pressing a button on the steering wheel. Patches were presented randomly every 5-10 seconds in the threshold experiment and every 6-10 fixations in the main experiment. Given that that most viewers fixed their gaze on the lead vehicle, thus leading quite long fixation durations, we opted not to present the Gabor patch for the duration of a fixation. Instead, we presented the Gabor patches for a duration of $67 \mathrm{~ms}$.

\subsubsection{Driving Task}

During the testing phase, participants viewed the playback from the driving simulator. Participants were required to follow a lead vehicle in the center lane of a 3-lane highway, and were instructed to maintain a 50 meter gap from the lead vehicle while maintaining their position in the center lane. Difficulty in the driving task was manipulated by increasing or decreasing the variability in the lead vehicle's speed.

\subsubsection{Cognitive Load Manipulation}

Besides manipulating the speed of the lead vehicle, participants also completed a 2-back task in the dual-task condition. Every 3 seconds, one of 26 letters in the alphabet was selected at random. There was a $25 \%$ chance that each letter in the series would be a valid target. During single-task trials, participants drove without performing the 2-Back task. (See example video of the dual Nback + Gabor discrimination task here.)

\section{$5.2 \quad$ Results}

\subsubsection{Gabor Orientation Discrimination Perfor- mance as a Function of Cognitive Load}

As shown in Figure 5, we found significant effects of N-back cognitive load (single-task vs. dual-task) on viewers' Gabor orientation discrimination performance, which was significantly interfered with by the 2-back task $\left(F(1,11)=5.5, p=.004\right.$, Cohen's $f^{2}$ $=1.98)$. However, we did not find an interaction between cognitive load and retinal eccentricity, as predicted by the "tunnel vision" hypothesis (i.e., with performance in the visual periphery more affected than performance in central vision). Instead, performance at $10^{\circ}$ and $15^{\circ}$ eccentricity was equally affected by the
N-back cognitive load, consistent with the general interference hypothesis of UFOV impairment. We note, however, that a stronger test of the tunnel vision hypothesis would also include targets at foveal or parafoveal eccentricities (i.e., $\leq 5^{\circ}$ ). We also found no effect of driving difficulty levels on Gabor orientation discrimination $(F(1,11)=.7, p=.4)$, though the lead vehicle was generally in central vision, thus potentially constituting a foveal load.

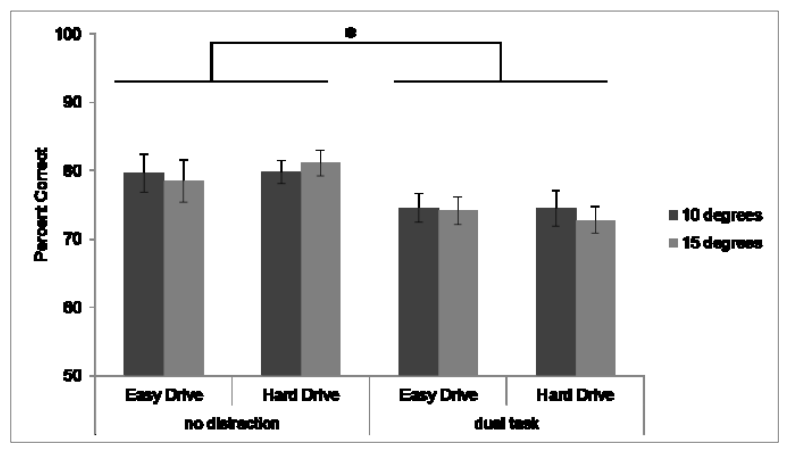

Figure 5. Gabor orientation discrimination as a function of single-versus dual-task (N-back), driving task difficulty, and retinal eccentricity of the Gabor patches.

\subsubsection{Driving Task Performance as a Function of Cognitive Load}

We evaluated lead vehicle following driving performance in terms of the standard deviation of following distance. As shown in Figure 6, following was more difficult with greater lead vehicle speed variability $(F(1,11)=11.9, p=.005)$. Importantly, however, following performance was unaffected by the 2-Back task $(F(1,11)=.01, p=.9)$. The driving task in this study was rela-

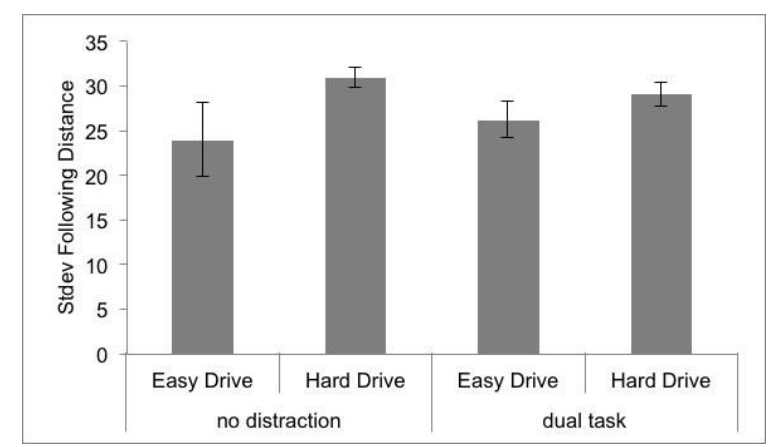

Figure 6. Driving task performance (SD of following distance) as a function of single-versus dual-task (N-back) and driving task difficulty.

tively simple, and it might be the case that a more demandingtask, such as a 3-Back task, is needed to disrupt following performance. Conversely, the fact that driving was unaffected by the N-back task while the Gabor orientation discrimination task was affected by the cognitive load manipulation indicates that our measure of attentional breadth is uniquely sensitive to changes in attentional resources.

\subsection{Discussion}

The results of our study with the Gabor orientation discrimination dependent measure of attention are very promising. Consistent with the underlying assumptions of our general framework for dynamically measuring the UFOV with gaze-contingent displays, 
we found a clear effect of cognitive load on our measure of attention. This suggests that discrimination tasks are more likely to be effective dependent measures of the UFOV than detection tasks within our general framework. In addition, regarding the effects of cognitive load on the UFOV, these results are more consistent with the general interference hypothesis than the tunnel vision hypothesis, although this conclusion remains tentative. Previous work has shown similar support for the general interference hypothesis when there is no foveal load, (i.e., no difficult task in central vision) [Crundall, et al. 2002, 1999; Williams 1988]. We had a demanding task in central vision, namely following a lead vehicle, but no Gabor patches presented in foveal vision, so further work will be needed to more rigorously test the tunnel vision hypothesis.

\section{$5 \quad$ Conclusions and Future Work}

We have described a new framework for measuring transitory changes in the UFOV in dynamic, realistic, simulated environments. The framework itself is relatively simple and flexible in terms of the parameters that can be manipulated by the experimenter. In particular, the framework uses gaze-contingent stimulus presentation [Duchowski, et al. 2004; Loschky, et al. 2002, 2000; McConkie, et al. 1976, 1975; Reingold, et al. 2003], so that stimuli can be presented dynamically while people naturally view scenes while nevertheless tightly controlling the retinal eccentricity of targets. Furthermore, by using adaptive threshold estimation for the stimuli at each target eccentricity, the targets are adjusted so that they are equally detectable at each retinal eccentricity under single-task conditions. Doing so allows us to unconfound 1) changes in the UFOV due to low-level eccentricity-dependent variations in visual sensitivity (e.g., acuity or contrast sensitivity) versus 2) changes in the UFOV due solely to the effects of attention.

In these studies, we have examined the utility of two different dependent measures of attention within our framework: blur detection and Gabor orientation discrimination. The results from these studies serve as a cautionary note that not all dependent measures are created equal, despite having tremendous control over our stimuli. In the blur detection task, we found no effect of cognitive load on blur sensitivity. This finding was replicated in two further experiments, which indicated that the null effects of cognitive load on blur detection could not be attributed to our research methods, but instead seems due to blur detection having minimal attentional demands [Loschky, et al. in press]. Conversely, the Gabor orientation task showed significant differences in performance under the cognitive load of 2-back N-back task. Thus, in using the general framework outlined here for dynamically measuring the UFOV, it is critical to first validate the measure of attention being used.

Finally, just as the dependent measure of attention is interchangeable with other measures, so too is the manipulation of attentional resources. While we have used the N-back task as a cognitive load manipulation in these experiments, other validated dual-task manipulations are also applicable (e.g. counting-backwards, mental math problems, etc.). Furthermore, we predict that other means of varying attentional resources can easily be included in our framework as well (e.g. fatigue, stress, or drug manipulations). In line with this idea, we will be continuing to explore the possible effects of driving difficulty manipulations on our dynamic measure of the UFOV.

Given the flexibility of our framework, we believe widespread applications of it would be useful for purposes of occupational screening and measuring changes in the UFOV during training or development. Although our current implementation of the framework using the Gabor orientation discrimination task is still being refined, we believe that the results reported here are broadly promising for gaze-contingent dynamic measures of transitory changes in the UFOV.

\section{Acknowledgments}

This work was supported by a grant from the Office of Naval Research (GRANT\# 10846128) to Lester Loschky.

\section{References}

Atchley, P. and Dressel, J. 2004. Conversation limits the functional field of view. Hum Factors, 46, 4, 664-673.

Ball, K. K., Beard, B. L., Roenker, D. L., Miller, R. L. and GRIGGS, D. S. 1988. Age and visual search: Expanding the useful field of view. Journal of the Optical Society of America, $5,12,2210-2219$.

Ball, K. K., Edwards, J. D., Ross, L. A. and McGwin, G. in press. Cognitive training decreases motor vehicle collision involvement in older drivers Journal of the American Geriatrics Society.

Ball, K. K., Owsley, C. and Beard, B. 1990. Clinical visual perimetry underestimates peripheral field problems in older adults. Clinical Vision Sciences, 5, 2, 113-125.

BurR, D. C. and WiJesundrA, S.-A. 1991. Orientation discrimination depends on spatial frequency. Vision Research, 31, 7, Äì, 1449-1452.

CARrasco, M. 2011. Visual attention: The past 25 years. Vision Research, 51, 13, 1484-1525.

Clay, O., Wadley, V., Edwards, J., Roth, D., Roenker, D. L. and BALL, K. K. 2005. Cumulative meta-analysis of the relationship between useful field of view and driving performance in older adults: Current and future implications. Optometry and vision science, 82, 8, 724-731.

Cohen, J. D., Perlstein, W. M., Braver, T. S., Nystrom, L. E., Noll, D. C., Jonides, J. and SMith, E. E. 1997. Temporal dynamics of brain activation during a working memory task. Nature, 386, 6625, 604-608.

Correa, Á., Lupianez, J., Milliken, B. and Tudela, P. 2004 Endogenous temporal orienting of attention in detection and discrimination tasks. Perception \& Psychophysics, 66, 2, 264 278.

Crundall, D. E., Underwood, G. and Chapman, P. R. 1999. Driving experience and the functional field of view. Perception, 28, 1075-1087.

Crundall, D. E., Underwood, G. and Chapman, P. R. 2002. Attending to the peripheral world while driving. Applied Cognitive Psychology, 16, 4, 459-475.

De Valois, R. L. and De Valois, K. K. 1988 Spatial vision. Oxford University Press, New York, NY, US. 
Duchowski, A. T., Bate, D., Stringfellow, P., Thakur, K., Melloy, B. J. and Gramopadhye, A. K. 2009. On spatiochromatic visual sensitivity and peripheral color lod management. Transactions on Applied Perception, 6, 2, 9:1-18.

Duchowski, A. T., Cournia, N. and Murphy, H. 2004. Gazecontingent displays: A review. CyberPsychology \& Behavior, $7,6,621-634$.

ENDSLEY, M. R. 1995. Toward a theory of situation awareness in dynamic-systems. Human Factors, 37, 1, 32-64.

Jaeggi, S. M., Buschkuehl, M., Perrig, W. J. and Meier, B. 2010. The concurrent validity of the n-back task as a working memory measure. Memory, 18, 4, 394-412.

Jahn, G., Oehme, A., Krems, J. F. and Gelau, C. 2005. Peripheral detection as a workload measure in driving: Effects of traffic complexity and route guidance system use in a driving study. Transportation research. Part E, Logistics and transportation review, 8, 3, 255-275.

KAERnBACH, C. 1990. A single-interval adjustment-matrix (siam) procedure for unbiased adaptive testing. The Journal of the Acoustical Society of America, 88, 6, 2645-2655.

Kane, M. J., Conway, A. R., Miura, T. K. and Colflesh, G. J. 2007. Working memory, attention control, and the n-back task: A question of construct validity. Journal of Experimental Psychology: Learning, Memory, and Cognition, 33, 3, 615-622.

Kenward, M. G. and Roger, J. H. 1997. Small sample inference for fixed effects from restricted maximum likelihood. Biometrics, 983-997.

Kontsevich, L. L. and Tyler, C. W. 1999. Bayesian adaptive estimation of psychometric slope and threshold. Vision Research, 39, 16, 2729-2737.

Kowler, E., Anderson, E., Dosher, B. and Blaser, E. 1995. The role of attention in the programming of saccades. Vision Research, 35, 13, 1897-1916.

LoschKy, L. C. and McConKIE, G. W. 2000 User performance with gaze contingent multiresolutional displays. ACM, City.

LoschKy, L. C. and McConKIE, G. W. 2002. Investigating spatial vision and dynamic attentional selection using a gazecontingent multi-resolutional display. Journal of Experimental Psychology: Applied, 8, 2, 99-117.

Loschky, L. C., McConkie, G. W., Yang, J. and Miller, M. E. 2005. The limits of visual resolution in natural scene viewing. Visual Cognition, 12, 6, 1057-1092.

Loschky, L. C., Ringer, R., Johnson, A., Larson, A. M., NeIDER, M. and KrAmer, A. in press. Blur detection is unaffected by cognitive load. Visual Cognition.
Loschky, L. C. and Wolverton, G. S. 2007. How late can you update gaze-contingent multiresolutioanl displays without detection? Transactions on Multimedia Computing, Communications, and Application, 3, 4:24, 1-10.

Mareschal, I. and ShaPley, R. M. 2004. Effects of contrast and size on orientation discrimination. Vision Research, 44, 1, 5767.

McConkie, G. W. and RAYNer, K. 1975. The span of the effective stimulus during a fixation in reading. Perception \& Psychophysics, 17, 6, 578-586.

MCCONKIE, G. W. and RAYNER, K. 1976 Identifying the span of the effective stimulus in reading: Literature review and theories of reading. International Reading Association, 137-162.

Murray, S. and Bex, P. J. 2010. Perceived blur in naturally contoured images depends on phase. Frontiers in psychology, 1.

Owen, A. M., McMillan, K. M., Laird, A. R. and Bullmore, E. 2005. N-back working memory paradigm: A meta-analysis of normative functional neuroimaging studies. Human Brain Mapping, 25, 1, 46-59.

Recarte, M. A. and NunEs, L. M. 2003. Mental workload while driving: Effects on visual search, discrimination, and decision making. Journal of Experimental Psychology-Applied, 9, 2, 119-137.

Reingold, E. M., Loschky, L. C., McConkie, G. W. and StAmpe, D. M. 2003. Gaze-contingent multi-resolutional displays: An integrative review. Human Factors, 45, 2, $307-$ 328 .

Roenker, D. L., Cissell, G. M., Ball, K. K., Wadley, V. G. and EDWARDS, J. D. 2003. Speed-of-processing and driving simulator training result in improved driving performance. Human Factors, 45, 2, 218-233.

Wang, B. and CiuffredA, K. J. 2004. Depth-of-focus of the human eye in the near retinal periphery. Vision Research, 44, $11,1115-1125$.

Webster, M. A., De Valois, K. K. and Switkes, E. 1990. Orientation and spatial-frequency discrimination for luminance and chromatic gratings. J. Opt. Soc. Am. A, 7, 6, 1034-1049.

Williams, L. J. 1985. Tunnel vision induced by a foveal load manipulation. Human Factors, 27, 2, 221-227.

Williams, L. J. 1988. Tunnel vision or general interference? Cognitive load and attentional bias are both important. American Journal of Psychology, 101, 171-191.

Xiao, J., Hays, J., Ehinger, K. A., Oliva, A. and Torralba, A. 2010. Sun database: Large-scale scene recognition from abbey to zoo. IEEE, 3485-3492. 